\title{
Perceived Ineffectiveness of Pharmacological Treatments for Dysmenorrhea
}

Chen X. Chen, PhD, ${ }^{1}$ Janet S. Carpenter, PhD, ${ }^{1}$ Michelle LaPradd, MS, ${ }^{2}$

Susan Ofner, MS, ${ }^{2}$ J. Dennis Fortenberry, MD, $\mathrm{MS}^{3}$

${ }^{1}$ Indiana University School of Nursing; ${ }^{2}$ Indiana University School of Medicine Department of

Biostatistics; ${ }^{3}$ ndiana University School of Medicine Department of Pediatrics

\section{Running Title:}

Ineffectiveness of Dysmenorrhea Treatments

Corresponding Author:

Chen X. Chen, 600 Barnhill Drive E415, Indianapolis, IN 46202

Email: cxchen@iu.edu, Tel: (317) 274-7441

Funding Disclosure: Dr. Chen is supported by the Grant Numbers KL2 TR002530 and UL1

TR002529 (A. Shekhar, PI) from the National Institutes of Health, National Center for Advancing Translational Sciences, Clinical and Translational Sciences Award and the EMPOWER Grant from Indiana University-Purdue University Indianapolis (C. Chen, PI). The content is solely the responsibility of the authors and does not represent the official views of the National Institutes of Health.

Conflict of Interest: Dr. Chen, Ms. LaPradd, Ms. Ofner, and Dr. Fortenberry have nothing to disclose. Outside the submitted work, Dr. Carpenter reports personal fees from RoundGlass Inc,

This is the author's manuscript of the work published in final form as:

Chen, C. X., Carpenter, J. S., LaPradd, M., Ofner, S., \& Fortenberry, J. D. (2020). Perceived Ineffectiveness of Pharmacological Treatments for Dysmenorrhea. Journal of Women's Health (2002). 
personal fees from Astellas Pharma Inc, personal fees from Kappa Sante, personal fees from Sojournix, and other from QUE oncology.

\begin{abstract}
Background: Dysmenorrhea affects most reproductive-age women. Common dysmenorrhea treatments vary in their effectiveness across individuals. Little is known about factors associated with perceived treatment ineffectiveness. The objectives of this study were to describe the perceived ineffectiveness of common pharmacological treatments for dysmenorrhea and investigate factors associated with perceived treatment ineffectiveness.

Methods: In this cross-sectional study, 678 women with dysmenorrhea (aged 14 to 42) provided data on perceived treatment ineffectiveness, dysmenorrhea symptom-based phenotypes, demographics, clinical factors, and psycho-behavioral characteristics. We used Fisher's exact tests to compare treatment ineffectiveness across three symptom-based phenotypes. We used logistic regressions to explore associations of phenotype, demographic, clinical, and psycho-behavioral correlates of perceived treatment ineffectiveness.
\end{abstract}

Results: Percentages perceiving treatments as ineffective were: $29.3 \%$ to $35.6 \%$ nonsteroidal anti-inflammatory drugs, $49.9 \%$ acetaminophen, 39.3\% combined oral contraceptive pills (OCPs). Factors associated with perceived ineffectiveness varied across treatments and included symptom-based phenotypes, clinical, and psycho-behavioral factors. For ibuprofen and acetaminophen, women with severe (vs. mild) pain phenotype and higher number of chronic pain conditions were more likely to perceive the treatments as ineffective. For OCPs, women with severe pain (vs. mild) phenotype, comorbid gynecological condition, less anxiety, and worse depressive symptoms were more likely to perceive the treatment as ineffective. Conclusion: A significant percentage of women reported ineffectiveness of dysmenorrhea treatments. Phenotypes, clinical, and psycho-behavioral factors were associated with treatment 
ineffectiveness. Future research should test if symptom-based phenotypes are associated with treatment effectiveness in clinical trials and investigate other factors that affect dysmenorrhea treatment effectiveness so treatments can be tailored to individuals.

Keywords: Dysmenorrhea, Pain, Pelvic Pain, Chronic Pain, Treatment Outcome Introduction

Dysmenorrhea is a chronic pain condition that affects $45 \%$ to $95 \%$ of reproductive-age women. ${ }^{1,2}$ Dysmenorrhea is characterized by abdominal cramps just before or during menstruation. Women with dysmenorrhea also may experience low back pain, headaches, and gastrointestinal symptoms. ${ }^{3}$ On a recurrent basis, dysmenorrhea negatively affects women's physical activities, sleep, and quality of life ${ }^{1}$ In the European Union, dysmenorrhea results in 3.6 million quality-adjusted life years lost annually, which is comparable to losses from chronic diseases such as type 1 diabetes, asthma, or chronic migraine. ${ }^{4}$ Dysmenorrhea also can interfere with work and school. Among young women globally, $10 \%$ to $46 \%$ miss one or more days of school each month due to dysmenorrhea. ${ }^{2,3,5}$

While the short-term impact of dysmenorrhea on women's lives is apparent, the longterm impact of dysmenorrhea is less well-known among researchers and clinicians. Dysmenorrhea may increase women's risk of developing other chronic pain conditions. For example, dysmenorrhea is a risk factor for irritable bowel syndrome (IBS) ${ }^{6}$ and non-cyclic pelvic pain. ${ }^{7}$ In imaging studies, dysmenorrhea has been associated with structural and functional changes in the brain, which may make affected women more susceptible to developing other chronic pain conditions later in life..$^{8-10}$ Given the connections between dysmenorrhea and other chronic pain conditions, treating dysmenorrhea early and effectively is more important than previously recognized. ${ }^{11}$

Although evidence-based pharmacological treatments are available for dysmenorrhea, their effectiveness varies among women. Cochrane reviews suggest that nonsteroidal antiinflammatory drugs (NSAIDs, e.g., ibuprofen, naproxen) and combined oral contraceptives pills 
(OCPs) are effective dysmenorrhea treatments. ${ }^{12,13}$ However, on the individual level, NSAIDs were ineffective for $18 \%$ of women with dysmenorrhea. ${ }^{14,15}$

Two gaps in the literature create barriers for effective management of dysmenorrhea.

First, the effectiveness or ineffectiveness rate for non-NSAIDs dysmenorrhea treatments, including OCPs, are rarely reported. Research using qualitative data suggests that some women with dysmenorrhea respond well to OCPs, while others find OCPs ineffective for menstrual pain. ${ }^{16}$ Little quantitative data are available regarding the perceived ineffectiveness of non-NSAIDs dysmenorrhea treatments.

Second, little is known about factors that are associated with the perceived ineffectiveness of dysmenorrhea pharmacological treatments. In other chronic pain conditions, symptom characteristics (e.g., types of symptoms) can affect treatment effectiveness. ${ }^{17}$ Symptom-based phenotypes have been identified in dysmenorrhea, including "mild localized pain" phenotype (characterized by mild abdominal cramps), "severe localized pain" phenotype (characterized by severe abdominal cramps), and "multiple severe symptoms" phenotype (characterized by pain at multiple sites and gastrointestinal symptoms)." ${ }^{18}$ The dysmenorrhea symptom-based phenotypes may be related to perceived treatment effectiveness. In addition, like other chronic pain conditions, ${ }^{19-23}$ demographic, clinical, and psycho-behavioral factors may explain variations in perceived treatment effectiveness/ineffectiveness in dysmenorrhea.

Therefore, the purpose of the study was to (1) describe perceived ineffectiveness of common pharmacological treatments for dysmenorrhea; (2) test whether perceived treatment ineffectiveness differs by dysmenorrhea symptom-based phenotypes; and (3) investigate phenotypic, demographic, clinical, and psycho-behavioral correlates of perceived treatment ineffectiveness. We hypothesized that perceived treatment ineffectiveness would be associated with dysmenorrhea symptom-based phenotypes and other factors.

\section{Materials and Methods}

\section{Design and Participants}


This was a cross-sectional descriptive study. Eligibility criteria were (a) female, (b) aged 14-42, (c) living in the United States, (d) able to read and write English, and (d) self-identified as having had dysmenorrhea symptoms in the last 6 months. We recruited participants from online survey panels maintained by Qualtrics (Qualtrics, Provo, UT). The panel provider used registrants' demographic data to identify and email invitations to potential participants.

\section{Procedures}

The university institutional review board approved the study. The survey panel provider emailed potential participants about the study opportunity. For those interested in participating, they proceeded by clicking the hyperlink to the survey embedded in the e-mail message. Further screening of potential participants occurred, directing those eligible to the study information page (i.e., the implied consent form). Those who agreed to participate proceeded to the survey questionnaires.

\section{Measurement}

Demographic and Clinical Variables. We collected self-reported demographic and clinical data on comorbid chronic pain and gynecological conditions. For comorbid chronic pain conditions, participants received instructions to report if they had any of the following chronic pain conditions: back pain, irritable bowel syndrome, migraines, non-migraine headaches, fibromyalgia, neck pain, pelvic pain outside of the menstrual period, interstitial cystitis, or other chronic pain. To determine if participants had comorbid gynecological conditions, we asked participants to report whether a healthcare provider had ever diagnosed the participant with endometriosis, uterine fibroids, bacterial vaginosis, polycystic ovary syndrome, sexually transmitted disease, pelvic inflammatory disease, adenomyosis, and other gynecological conditions.

Perceived Treatment Effectiveness. We collected data on pharmacological treatments used by women with dysmenorrhea. ${ }^{24}$ Participants were asked to select pain medications they had ever used to prevent or treat menstrual pain from a checklist of pain medications. The 
checklist included ibuprofen, naproxen, acetaminophen alone, acetaminophen combined with caffeine and antihistamine, acetaminophen combined with antihistamine and diuretic, opioid, and other pain medications. We provided examples of common brand names to ensure comprehension. For each pain medication they selected, participants received instructions to respond to the follow-up question, "How effective was [insert the selected pain medication] to manage your menstrual pain?" The five response options were not effective at all, a little effective, moderately effective, quite effective, and extremely effective.

Similarly, participants were asked to select hormonal contraceptives they had ever used to prevent or treat menstrual pain from a checklist of hormonal contraceptives. The checklist included combined oral contraceptive pill, progestin-only pill (minipill), hormonal intrauterine device (IUD), skin patch, medroxyprogesterone acetate injection (shot), implant, and estrogen and progestin ring (ring). Examples of common brand names were provided to ensure comprehension. For each hormonal contraceptive they selected, participants received a prompt to respond to the follow-up question, "How effective was [insert the selected hormonal contraceptive] to manage your menstrual pain?" The five response options were not effective at all, a little effective, moderately effective, quite effective, and extremely effective.

For Aims 2 and 3, we dichotomized the five response options into "perceived effective" and "perceived ineffective." Specifically, we combined categories of "extremely effective," "quite effective," and "moderately effective" into a category of "perceived effective." We combined categories of "a little effective" and "not at all effective" into a category of "perceived ineffective." For statistical purposes, dichotomization allows for larger counts in outcome categories and intuitive interpretation.

Symptom-based phenotypes. Participants rated the severity of 14 dysmenorrhearelated symptoms in their most recent menstrual cycle: abdominal cramps, dull abdominal pain or discomfort, low back pain, pain in the upper thighs, headache or migraines, pain when the bladder was full, aches all over, bloating, nausea, vomiting, diarrhea (loose stools), constipation 
(hard stools), more bowel movements than usual, and fewer bowel movements than usual. ${ }^{18,25}$ Participants rated the severity of each symptom on a 0 ("not present") to 10 ("extremely severe") scale. Each severity rating was then grouped into one of four severity categories based on established cut points: no symptom (0), mild (1-4), moderate (5-6), and severe (7-10). ${ }^{26,27}$ Then, we conducted a latent class analysis of the symptom severity data. Consistent with previous research, ${ }^{18}$ we identified three symptom-based phenotypes, including "mild localized pain" phenotype, "severe localized pain" phenotype, and "multiple severe symptoms" phenotype. ${ }^{18}$ Based on probabilities, we grouped individual participants into one of the three dysmenorrhea symptom-based phenotypes.

Psycho-behavioral Variables. We assessed psycho-behavioral symptoms, including pain catastrophizing, perceived stress, anxiety, depression, and sleep disturbance.

Pain catastrophizing is defined as a set of negative thoughts related to pain experiences, including rumination, magnification, and helplessness. ${ }^{28}$ We measured pain catastrophizing with the Pain Catastrophizing Scale. ${ }^{29}$ The 13 -item scale measures the extent to which participants worry, amplify, and feel helpless about the experience of pain on a 5-point (0 to 4$)$ scale. Total scores range from 0 to 52, with higher scores indicating greater pain catastrophizing. The Pain Catastrophizing Scale is reliable and valid in clinical and non-clinical samples. ${ }^{29}$

Perceived stress was measured by the Perceived Stress Scale, ${ }^{30}$ a classic, wellvalidated stress assessment tool. Participants rate each of the 10 items on a 5-point (0-4) scale; the total score ranges from 0-40, with higher scores indicating higher perceived stress.

Anxiety was measured by the reliable and valid PROMIS Anxiety 8-item Short Form (8a). ${ }^{31}$ The PROMIS Anxiety instrument assesses self-reported fear, anxiety, and tension. Participants rated the severity of anxiety symptoms in the past seven days using a 5-point (1-5) response scale.

Depression was measured by the reliable and valid 8-item PROMIS Depression Short Form (8b). ${ }^{31}$ "The PROMIS Depression Short Form assesses self-reported mood, views of self, 
social cognition, affect, and engagement over the past seven days. Participants rated the severity of their depression using a 5-point (1-5) response scale.

Sleep disturbance was measured by the reliable and valid PROMIS Sleep Disturbance 8-item Short Form (8b). ${ }^{32,33}$ Participants rated the severity of sleep disturbance during the past seven days using a 5-point (1-5) scale.

For the three PROMIS scales discussed above, raw scale scores (summed item responses range from 8 to 40 ) were converted to T-scores using a conversion table. A T-score of 50 is the average for the United States general population with a standard deviation of 10 . A higher PROMIS T-score indicates more of the concept being measured (i.e., severer depression, anxiety, and sleep disturbance). More information can be found at www.healthmeasures.net.

\section{Data Analysis}

We used descriptive statistics to summarize participants' demographic, clinical, and psycho-behavioral characteristics. To summarize women's perceived ineffectiveness of common pharmacological dysmenorrhea treatments (Aim 1), we used descriptive statistics, specifically counts and frequencies. For a given individual, we counted a treatment as ineffective when the participant rated it as "not at all effective" or "a little effective."

To test whether perceived treatment ineffectiveness differed by dysmenorrhea symptombased phenotypes (Aim 2), we used Fisher's exact test. Fisher's exact test was chosen since the expected frequencies for less frequently used treatments were smaller than 5 .

We evaluated factors associated with perceived treatment ineffectiveness (Aim 3) for three treatments: ibuprofen, acetaminophen, and combined OCP. These treatments were selected because at least $25 \%$ of the study sample reported using them. The outcome reference group was those who perceived a specific treatment as effective. Bivariate models of each of the three treatments were fit with symptom-based phenotypes, demographic, clinical, 
and psycho-behavioral characteristics as explanatory variables. More specifically, independent variables were phenotypes, demographic factors (age, race, ethnicity), clinical factors (number of comorbid pain conditions, the existence of a comorbid gynecological condition), and psychobehavioral factors (pain catastrophizing, perceived stress, anxiety, depression, and sleep disturbance). Missingness was assessed to determine if any of the variables considered for modeling had large amounts of missing data. Across variables, missing data rates ranged from $0 \%$ to $1 \%$ of the cases and were acceptable.

We built multiple logistic regression models for each treatment by fitting models to each possible combination of the 12 explanatory variables. These variables included age, two dummy variables for phenotypes, three dummy variables for race, ethnicity, the number of comorbid pain conditions, existence of a comorbid gynecological condition, pain catastrophizing, perceived stress, anxiety, depression, and sleep disturbance. We selected the model with the smallest Akaike's Information Criterion (AIC) as the best fitting model. This variable selection approach has been shown to be consistent, where consistency is defined in terms of model selection. ${ }^{34}$ Correlations were assessed between all the independent variables of interest for the model to check for multicollinearity. Also, the variance inflation factors (VIFs) for all three models were below three, indicating that multicollinearity between the explanatory variables was not a concern.

The type I error rate was set at a 0.05 level for each test. Statistical analyses were performed using the $\mathrm{R}$ package.

\section{Results}

\section{Sample Characteristics}

The mean age of the sample $(\mathrm{N}=678)$ was 28.0 years $(S D=7.6$; range $=14-42)$. Most were White (67.7\%), 13.3\% were Black/African American, $7.8 \%$ were Asian, and $11.2 \%$ were other (including American Indian or Alaska Native, Native Hawaiian or Pacific Islander, and other). Most participants were non-Hispanic/non-Latino (87.9\%). 
Among participants, more than half $(57.7 \%)$ had another chronic pain condition, including low back pain $(31.7 \%)$, migraine headaches $(28.8 \%)$, neck pain $(13.9 \%)$, non-migraine headaches (10.2\%), pelvic pain occurring outside of menstrual period $(9.7 \%)$, and irritable bowel syndrome (8.3\%). About one-fourth of participants $(25.2 \%)$ were previously diagnosed with another gynecological condition, such as bacterial vaginosis $(9.1 \%)$, endometriosis $(4.9 \%)$, polycystic ovary syndrome (4.9\%), and uterine fibroids (3.1\%). The mean score was 18.3 $(S D=12.8)$ for pain catastrophizing, $22.4(S D=6.4)$ for perceived stress, $62.3(S D=8.8)$ for anxiety, $57.1(\mathrm{SD}=9.7)$ for depression, and $51.6(\mathrm{SD}=4.0)$ for sleep disturbance.

\section{Aim 1: Perceived Ineffectiveness of Dysmenorrhea Treatments}

Figure 1 and Table 1 shows treatments women used and perceived effectiveness/ ineffectiveness for each treatment. The three most common treatments that women tried were ibuprofen, acetaminophen, and combined OCP.

For commonly used pain medications, $29.3 \%$ of ibuprofen users rated it as ineffective, and $35.6 \%$ of naproxen users rated it as ineffective. For acetaminophen alone, $49.9 \%$ of users rated it ineffective. For acetaminophen combined with caffeine and antihistamine, $34.8 \%$ of users rated it as ineffective. For acetaminophen combined with antihistamine and diuretic, $51.7 \%$ of users rated it as ineffective. For opioids, $9.2 \%$ of users rated them as ineffective.

Among estrogen-progestin combined contraceptives, $39.3 \%$ of OCPs users, $48.4 \%$ of implant users, and $40 \%$ of estrogen and progestin ring users rated the treatment as ineffective. For progestin-only contraceptives, $46.8 \%$ of hormonal IUD users rated the treatment as ineffective, $40.8 \%$ of medroxyprogesterone acetate injection (i.e., shot) users rated the treatment as ineffective for dysmenorrhea, and $50.0 \%$ of progestin-only pill users rated the treatment as ineffective.

\section{Aim 2: Perceived Treatment Ineffectiveness by Dysmenorrhea Symptom-based}

\section{Phenotypes}


As shown in Table 1, perceived treatment ineffectiveness for ibuprofen, acetaminophen combined with caffeine and antihistamine, and combined OCP significantly differed across dysmenorrhea symptom-based phenotype groups (all $p<0.05$ ). Specifically, women in the "multiple severe symptoms" phenotype group had the highest rate of perceiving ibuprofen, combined OCPs, and acetaminophen combined with caffeine and antihistamine as ineffective.

\section{Aim 3: Phenotype, Demographic, Clinical, and Psycho-behavioral Correlates of Perceived}

\section{Treatment Ineffectiveness}

Ibuprofen. Table 2 shows the correlates of perceived ineffectiveness of ibuprofen from bivariate and multiple logistic regression models. In bivariate models, the likelihood of perceiving ibuprofen as ineffective was $117 \%$ higher in the "severe localized pain" phenotype than the "mild localized pain" phenotype $(\mathrm{OR}=2.17,95 \% \mathrm{Cl} 1.32-3.59), 128 \%$ higher in the "multiple severe symptoms" phenotype than the "mild localized pain" phenotype (OR=2.28, 95\% Cl 1.34-3.85), $35 \%$ higher with every additional comorbid pain condition (OR=1.35, 95\% Cl 1.18-1.55), and $2 \%$ higher with every point increase in pain catastrophizing $(\mathrm{OR}=1.02,95 \% \mathrm{Cl} 1.01-1.04)$. In the best fit multiple logistic regression model for ibuprofen, the odds of perceiving ibuprofen as ineffective was $92 \%$ higher for the "severe localized pain" phenotype group $(\mathrm{OR}=1.92,95 \% \mathrm{Cl}$ 1.13-3.25) and $26 \%$ higher for each additional chronic pain condition $(\mathrm{OR}=1.26,95 \% \mathrm{Cl} 1.08$ 1.47).

Acetaminophen. Table 3 shows the correlates of perceived ineffectiveness of acetaminophen from bivariate and multiple logistic regression models. In the bivariate models, the likelihood of perceiving acetaminophen as ineffective was $83 \%$ higher in the "severe localized pain" phenotype than the "mild localized pain" phenotype $(\mathrm{OR}=1.83,95 \% \mathrm{Cl} 1.09$ $3.07), 3 \%$ higher for each year increase in age (OR=1.03, 95\% $\mathrm{Cl} 1.00-1.07), 73 \%$ lower among Asian users (OR=0.27, 95\% Cl .09-.83), 21\% higher for each additional comorbid pain condition $(\mathrm{OR}=1.21,95 \% \mathrm{Cl} 1.04-1.41)$, and $1 \%$ higher for every one point increase in pain catastrophizing $(\mathrm{OR}=1.01,95 \% \mathrm{Cl} 1.00-1.02)$. In the best fit multiple logistic regression model 
for acetaminophen, the odds of perceived ineffectiveness were $72 \%$ higher in the "severe localized pain" phenotype $(\mathrm{OR}=1.72,95 \% \mathrm{Cl} 1.01-2.91)$ and $21 \%$ higher for each additional chronic pain condition (OR=1.21, 95\% Cl 1.03-1.41).

Combined OCPs. Table 4 shows the correlates of perceived ineffectiveness of combined OCPs from bivariate and multiple logistic regression models. In the bivariate models, the likelihood of perceiving OCPs as ineffective was $263 \%$ higher in the "severe localized pain" phenotype compared to the "mild localized pain" phenotype (OR=3.63, 95\% Cl 1.74-7.55), 22\% higher with each additional chronic pain condition (OR=1.22, 95\% Cl 1.02-1.47), and 161\% higher for individuals with a comorbid gynecological condition compared to those without $(\mathrm{OR}=2.61,95 \% \mathrm{Cl} 1.43-4.78)$. In the best fit multiple logistic regression model for OCPs, the odds of perceived ineffectiveness were $246 \%$ higher in the "severe localized pain" phenotype $(\mathrm{OR}=3.46,95 \% \mathrm{Cl} 1.58-7.57), 156 \%$ higher for individuals with a comorbid gynecological condition compared to those without (OR=2.56, 95\% Cl 1.34-4.89), 6\% lower for every one point increase in anxiety score $(\mathrm{OR}=0.94,95 \% \mathrm{Cl} 0.89-1.00)$, and $6 \%$ higher for every one point increase in depression score $(\mathrm{OR}=1.06,95 \% \mathrm{Cl} 1.00-1.03)$.

\section{Discussion}

In this study, we reported the perceived ineffectiveness of common pharmacological dysmenorrhea treatments. We tested whether perceived treatment ineffectiveness differed by symptom-based phenotypes, and explored phenotypes, demographic, clinical, and psychobehavioral correlates of treatment ineffectiveness. Consistent with previous research, ${ }^{15,16,}$ this study shows heterogeneity in perceived dysmenorrhea treatment effectiveness. The percentages perceiving NSAIDs as ineffective (i.e., 29.3\%-35.6\%) were higher than the 18\% reported in a review of clinical trials. ${ }^{35}$ The higher NSAIDs ineffectiveness rate reported in our study may be due to assessing a more heterogeneous sample compared to highly controlled clinical trials. For example, we included women who had comorbid gynecological conditions. We 
also included participants with chronic overlapping pain conditions, as recommended. ${ }^{36}$ Given the heterogeneity in treatment effectiveness, new and precision-based (i.e., tailored) dysmenorrhea treatments are needed. To optimize dysmenorrhea treatments, factors associated with perceived treatment effectiveness and ineffectiveness need to be further investigated.

This study is the first to compare perceived dysmenorrhea treatment ineffectiveness by symptom-based dysmenorrhea phenotypes. As we hypothesized, women in the more severe phenotype groups were less likely to perceive commonly used treatments as effective (NSAIDs, acetaminophen, and combined OCPs). We found that dysmenorrhea symptom-based phenotypes were significant predictors for the perceived ineffectiveness of ibuprofen, acetaminophen, and combined OCPs, even after controlling for other demographic, clinical, and psycho-behavioral factors. Findings suggest that certain dysmenorrhea symptom-based phenotypes may be more challenging to treat or more treatment-resistant. Given that the women in the "multiple severe symptoms" phenotype group experienced pain at multiple sites and severe gastrointestinal symptoms, it is likely that different, possibly central pain mechanisms may be involved, and thus different treatments may be needed. This "multiple severe symptoms" phenotype group may have a heightened sensitivity of the central nervous system, which would explain why its members are more likely to fail standard treatments. ${ }^{18}$ If the central mechanisms are significantly involved in the "multiple severe symptoms" phenotype, treatments targeting central mechanisms (e.g., tricyclic antidepressants, gabapentin, cognitive behavioral interventions, and exercises) ${ }^{37-39}$ may be promising.

For demographic correlates, although age and race were significant in bivariate models for acetaminophen only, we found no demographic correlates in multiple logistic regression models for any of the three treatments. Older women were more likely to perceive acetaminophen as ineffective. It is possible that aging increases the chances for more repeated menstrual pain episodes that sensitize the nervous system to pain, which makes dysmenorrhea 
more refractory to treatment. Longer number of years with dysmenorrhea has been associated with a more negative impact of dysmenorrhea on the central nervous system. ${ }^{8}$ Compared to Whites, Asians were less likely to report acetaminophen as an ineffective treatment for dysmenorrhea. A previous study suggested that Chinese participants had different acetaminophen pharmacokinetics from White participants, with Chinese participants absorbing acetaminophen more rapidly than White participants. ${ }^{40}$ The racial differences could also be due to psycho-behavioral (e.g., treatment preferences, family influences), social, and cultural factors. For clinical correlates, the number of comorbid pain conditions was significant in bivariate models for all three treatments. After controlling for other covariates, comorbid pain conditions increased the odds of perceiving ibuprofen or acetaminophen as ineffective. In other words, with each additional chronic pain condition, women with dysmenorrhea were more likely to be refractory to common over-the-counter pain treatments. This may be a consequence of central pain regulatory systems disruption. ${ }^{36}$ Women with dysmenorrhea and comorbid chronic pain may require more intensive treatments or new treatments. In this study, women with a comorbid gynecological condition were more likely to perceive combined OCPs as ineffective. It is possible that certain gynecological conditions contribute to treatment resistance. It is also possible that women who do not respond to combined OCPs as a dysmenorrhea treatment were more likely to receive a diagnosis of a gynecological condition, such as endometriosis. Additional research on these relationships is warranted.

For psycho-behavioral factors, pain catastrophizing was significant in bivariate models for two of the three treatments (ibuprofen and acetaminophen). However, the effect size values (i.e., odds ratios) were very small. In addition, pain catastrophizing was not a significant predictor in the multiple regression models. Interestingly, for the multiple logistic regression model on combined OCPs, higher depression was associated with perceiving combined OCPs as ineffective, while higher anxiety was associated with perceiving combined OCPs as effective. It is unclear why depression and anxiety had an inverse relationship with the perceived 
ineffectiveness of OCPs for treating dysmenorrhea. We conducted a post-hoc analysis to check the potential interaction effect of depression and anxiety on the perceived ineffectiveness of combined OCPs, but it was not significant $(p=0.33)$. When the interaction term was added, however, anxiety and depression no longer had an inverse relationship with perceived ineffectiveness. For some women with dysmenorrhea, depression may increase somatic symptoms, which heightens the risk of developing other chronic pain conditions ${ }^{41}$ and contributes to ineffectiveness of pharmacological treatments. Research (as summarized in a review $)^{42}$ has suggested that individuals with more depressive symptoms were less likely to respond to pain treatments. The complex relationship between OCPs and depression ${ }^{43-45}$ also may impact OCPs' perceived effectiveness/ineffectiveness in dysmenorrhea. To our knowledge, little is known about the impact of anxiety on pharmacological pain treatment outcomes. Additional research on these relationships is warranted.

This study had limitations. First, there were small numbers of users for certain treatments (e.g., opioids, progestin-only pills, patch, shot). The perceived effectiveness/ ineffectiveness data for these treatments need to be interpreted with caution. Second, we did not assess other factors that could impact treatment ineffectiveness, including pathological factors (e.g., severity of endometriosis), pharmacogenetics, and medication dosing. ${ }^{15}$ Research has suggested that drug dosage was retrospectively recalled by research participants less accurately than drug names. ${ }^{46,47}$ Longitudinal study designs will be required for future research on the effect of medication dosing on treatment effectiveness/ineffectiveness. Third, there could be recall bias and self-report bias related to survey responses. In addition, participants might recall treatment effectiveness in different ways (e.g., some may have considered certain times when treatments were effective/ineffective rather than considering the overall effectiveness/ineffectiveness of treatments over longer spans of time). Fourth, the crosssectional design prevents conclusions about causality. It is possible that certain covariates (e.g., symptom-based phenotypes and psycho-behavioral factors) reflect the 
effectiveness/ineffectiveness of treatments rather than lead to different treatment outcomes. Fifth, as we intended to limit the length of our survey, we did not investigate the perceived effectiveness/ineffectiveness of non-pharmacological treatments. Previous research suggests that women use a large variety of complementary health approaches and non-pharmacological strategies (e.g., heat, dietary supplements) for dysmenorrhea. ${ }^{24,48,49}$ Factors that affect their differential effectiveness among women need to be investigated in the future.

Despite these limitations, there are implications for future research. First, in clinical trials evaluating dysmenorrhea treatments, researchers can test if symptom-based dysmenorrhea phenotypes are associated with treatment responsiveness. Second, additional research is needed to explore factors that may explain heterogeneity in treatment effectiveness. Such research is necessary to develop precision-based dysmenorrhea treatments. Third, additional research could evaluate whether treatments that target the central nervous system (e.g., tricyclic antidepressants and gabapentin) in the "multiple severe symptoms" phenotype group are effective. If positive, findings could provide confirmation of central nervous system involvement underlying the pain. ${ }^{18}$

\section{Conclusion}

A significant percentage of women perceived common pharmacological dysmenorrhea treatments as ineffective. Phenotypes, clinical, and psycho-behavioral factors were associated with treatment ineffectiveness. Future research should test if symptom-based phenotypes are associated with treatment effectiveness in clinical trials and investigate other factors that affect treatment effectiveness, so that dysmenorrhea treatments can be tailored to individuals.

Acknowledgments: The authors thank Tabitha Murphy, RN, BSN for assistance with data collection, Peter Krombach, MPH for supporting data management, and Tasneem Talib, PhD for editorial suggestions. Dr. Chen was supported by the Grant Numbers KL2 TR002530 and UL1 TR002529 (A. Shekhar, PI) from the National Institutes of Health, National Center for Advancing 
Translational Sciences, Clinical and Translational Sciences Award and the EMPOWER Grant from Indiana University-Purdue University Indianapolis (C. Chen, PI). The content is solely the responsibility of the authors and does not represent the official views of the National Institutes of Health.

Author Disclosure Statement: Dr. Chen, Ms. LaPradd, Ms. Ofner, and Dr. Fortenberry have nothing to disclose. Dr. Carpenter reports personal fees from RoundGlass Inc., personal fees from Astellas Pharma Inc., personal fees from Kappa Sante, personal fees from Sojournix, and other from QUE oncology, outside the submitted work. 


\section{References}

1. lacovides S, Avidon I, Baker FC. What we know about primary dysmenorrhea today: a critical review. Hum Reprod Update. 2015;21(6):762-778.

2. Klein JR, Litt IF. Epidemiology of adolescent dysmenorrhea. Pediatrics. 1981;68(5):661-664.

3. Dawood MY. Nonsteroidal Anti-Inflammatory Drugs and Changing Attitudes toward Dysmenorrhea. American Journal of Medicine. 1988;84(5a):23-29.

4. Rencz F, Pentek M, Stalmeier PFM, et al. Bleeding out the quality-adjusted life years: evaluating the burden of primary dysmenorrhea using time trade-off and willingness-to-pay methods. Pain. 2017;158(11):2259-2267.

5. O'Connell K, Davis AR, Westhoff C. Self-treatment patterns among adolescent girls with dysmenorrhea. J Pediatr Adolesc Gynecol. 2006;19(4):285-289.

6. Olafsdottir LB, Gudjonsson H, Jonsdottir HH, Bjornsson E, Thjodleifsson B. Natural history of irritable bowel syndrome in women and dysmenorrhea: a 10-year follow-up study. Gastroenterol Res Pract. 2012;2012:534204.

7. Westling AM, Tu FF, Griffith JW, Hellman KM. The association of dysmenorrhea with noncyclic pelvic pain accounting for psychological factors. American journal of obstetrics and gynecology. 2013;209(5):422.e421-422.e410.

8. Vincent K, Warnaby C, Stagg CJ, Moore J, Kennedy S, Tracey I. Dysmenorrhoea is associated with central changes in otherwise healthy women. Pain. 2011;152(9):1966-1975.

9. Wei SY, Chao HT, Tu CH, et al. Changes in functional connectivity of pain modulatory systems in women with primary dysmenorrhea. Pain. 2016;157(1):92-102.

10. Tu CH, Niddam DM, Chao HT, et al. Brain morphological changes associated with cyclic menstrual pain. Pain. 2010;150(3):462-468.

11. Berkley KJ. Primary dysmenorrhea: An urgent mandate. Pain: Clinical Updates 2013;21(3):1-7.

12. Marjoribanks J, Ayeleke RO, Farquhar C, Proctor M. Nonsteroidal anti-inflammatory drugs for dysmenorrhoea. Cochrane Database Syst Rev. 2015(7):Cd001751.

13. Wong CL, Farquhar C, Roberts H, Proctor M. Oral contraceptive pill for primary dysmenorrhoea. Cochrane Database Syst Rev. 2009(4):Cd002120.

14. Lundstrom V, Green K. Endogenous levels of prostaglandin F2alpha and its main metabolites in plasma and endometrium of normal and dysmenorrheic women. Am J Obstet Gynecol. 1978;130(6):640-646.

15. Oladosu FA, Tu FF, Hellman KM. Nonsteroidal antiinflammatory drug resistance in dysmenorrhea: epidemiology, causes, and treatment. Am J Obstet Gynecol. 2018;218(4):390-400.

16. Chen CX, Draucker CB, Carpenter JS. What women say about their dysmenorrhea: a qualitative thematic analysis. BMC Womens Health. 2018;18(1):47.

17. Wheeler SD. Phenotype-driven preventive strategies for migraine and other headaches. Neurologist. 2009;15(2):59-70.

18. Chen CX, Ofner S, Bakoyannis G, Kwekkeboom KL, Carpenter JS. Symptoms-Based Phenotypes Among Women With Dysmenorrhea: A Latent Class Analysis. West J Nurs Res. 2018;40(10):14521468.

19. Brummett CM, Urquhart AG, Hassett AL, et al. Characteristics of fibromyalgia independently predict poorer long-term analgesic outcomes following total knee and hip arthroplasty. Arthritis Rheumatol. 2015;67(5):1386-1394.

20. Schiano di Cola F, Caratozzolo S, Liberini P, Rao R, Padovani A. Response Predictors in Chronic Migraine: Medication Overuse and Depressive Symptoms Negatively Impact Onabotulinumtoxin-A Treatment. Front Neurol. 2019;10:678.

21. Hancock MJ, Maher CG, Latimer J, McLachlan AJ, Day RO, Davies RA. Can predictors of response to NSAIDs be identified in patients with acute low back pain? Clin J Pain. 2009;25(8):659-665.

22. Manfredini D, Favero L, Del Giudice A, Masiero S, Stellini E, Guarda-Nardini L. Axis II psychosocial findings predict effectiveness of TMJ hyaluronic acid injections. Int J Oral Maxillofac Surg. 2013;42(3):364-368.

23. Walsh TM, LeBlanc L, McGrath PJ. Menstrual pain intensity, coping, and disability: the role of pain catastrophizing. Pain Med. 2003;4(4):352-361. 
24. Chen CX, Kwekkeboom KL, Ward SE. Beliefs about dysmenorrhea and their relationship to selfmanagement. Research in Nursing \& Health. 2016;39(4):263-276.

25. Chen CX, Kwekkeboom KL, Ward SE. Self-report pain and symptom measures for primary dysmenorrhoea: a critical review. Eur J Pain. 2015;19(3):377-391.

26. Serlin RC, Mendoza TR, Nakamura Y, Edwards KR, Cleeland CS. When is cancer pain mild, moderate or severe? Grading pain severity by its interference with function. Pain. 1995;61(2):277-284.

27. Oldenmenger WH, de Raaf PJ, de Klerk C, van der Rijt CC. Cut points on 0-10 numeric rating scales for symptoms included in the Edmonton Symptom Assessment Scale in cancer patients: a systematic review. J Pain Symptom Manage. 2013;45(6):1083-1093.

28. Sullivan MJ, Thorn B, Haythornthwaite JA, et al. Theoretical perspectives on the relation between catastrophizing and pain. Clin J Pain. 2001;17(1):52-64.

29. Sullivan MJL, Bishop SR, Pivik J. The Pain Catastrophizing Scale: Development and validation. Psychological Assessment. 1995;7(4):524-532.

30. Cohen S, Kamarck T, Mermelstein R. A global measure of perceived stress. J Health Soc Behav. 1983;24: 385-396.

31. Schalet BD, Pilkonis PA, Yu L, et al. Clinical validity of PROMIS Depression, Anxiety, and Anger across diverse clinical samples. Clin Epidemiol. 2016;73:119-127.

32. $\mathrm{Yu}$ L, Buysse DJ, Germain A, et al. Development of short forms from the PROMIS sleep disturbance and Sleep-Related Impairment item banks. Behav Sleep Med. 2011;10(1):6-24.

33. Buysse DJ, Yu L, Moul DE, et al. Development and validation of patient-reported outcome measures for sleep disturbance and sleep-related impairments. Sleep. 2010;33(6):781-792.

34. Shao J. An asymptotic theory for linear model selection. Statistica Sinica. 1997;7(2):221-242.

35. Owen PR. Prostaglandin synthetase inhibitors in the treatment of primary dysmenorrhea. Outcome trials reviewed. Am J Obstet Gynecol. 1984;148(1):96-103.

36. Maixner W, Fillingim RB, Williams DA, Smith SB, Slade GD. Overlapping Chronic Pain Conditions: Implications for Diagnosis and Classification. J Pain. 2016;17(9 Suppl):T93-T107.

37. Ellingson LD, Stegner AJ, Schwabacher IJ, Koltyn KF, Cook DB. Exercise Strengthens Central Nervous System Modulation of Pain in Fibromyalgia. Brain Sci. 2016;6(1):8.

38. Woolf CJ. Central sensitization: Implications for the diagnosis and treatment of pain. Pain. 2011;152(3 Suppl):S2-15.

39. Salomons TV, Moayedi M, Erpelding N, Davis KD. A brief cognitive-behavioural intervention for pain reduces secondary hyperalgesia. Pain. 2014;155(8):1446-1452.

40. Critchley JA, Critchley LA, Anderson PJ, Tomlinson B. Differences in the single-oral-dose pharmacokinetics and urinary excretion of paracetamol and its conjugates between Hong Kong Chinese and Caucasian subjects. J Clin Pharm Ther. 2005;30(2):179-184.

41. Zuckerman RM, Silton RL, Tu FF, Eng JS, Hellman KM. Somatic symptoms in women with dysmenorrhea and noncyclic pelvic pain. Arch Womens Ment Health. 2018;21(5):533-541.

42. Bair MJ, Robinson RL, Katon W, Kroenke K. Depression and pain comorbidity: a literature review. Arch Intern Med. 2003;163(20):2433-2445.

43. Joffe H, Cohen LS, Harlow BL. Impact of oral contraceptive pill use on premenstrual mood: predictors of improvement and deterioration. Am J Obstet Gynecol. 2003;189(6):1523-1530.

44. Skovlund CW, Mørch LS, Kessing LV, Lidegaard $\varnothing$. Association of Hormonal Contraception With Depression. JAMA Psychiatry. 2016;73(11):1154-1162.

45. Keyes KM, Cheslack-Postava K, Westhoff C, et al. Association of hormonal contraceptive use with reduced levels of depressive symptoms: a national study of sexually active women in the United States. Am J Epidemiol. 2013;178(9):1378-1388.

46. West SL, Savitz DA, Koch G, Strom BL, Guess HA, Hartzema A. Recall accuracy for prescription medications: self-report compared with database information. Am J Epidemiol. 1995;142(10):11031112.

47. Lewis JD, Strom BL, Kimmel SE, et al. Predictors of recall of over-the-counter and prescription nonsteroidal anti-inflammatory drug exposure. Pharmacoepidemiol Drug Saf. 2006;15(1):39-45.

48. Campbell MA, McGrath PJ. Non-pharmacologic strategies used by adolescents for the management of menstrual discomfort. Clin J Pain. 1999;15(4):313-320. 
49. Chen CX, Groves D, Miller WR, Carpenter JS. Big Data and Dysmenorrhea: What Questions Do Women and Men Ask About Menstrual Pain? J Womens Health 2018;27(10):1233-1241. 
Figure 1. Perceived Ineffectiveness and Effectiveness of Dysmenorrhea Treatments

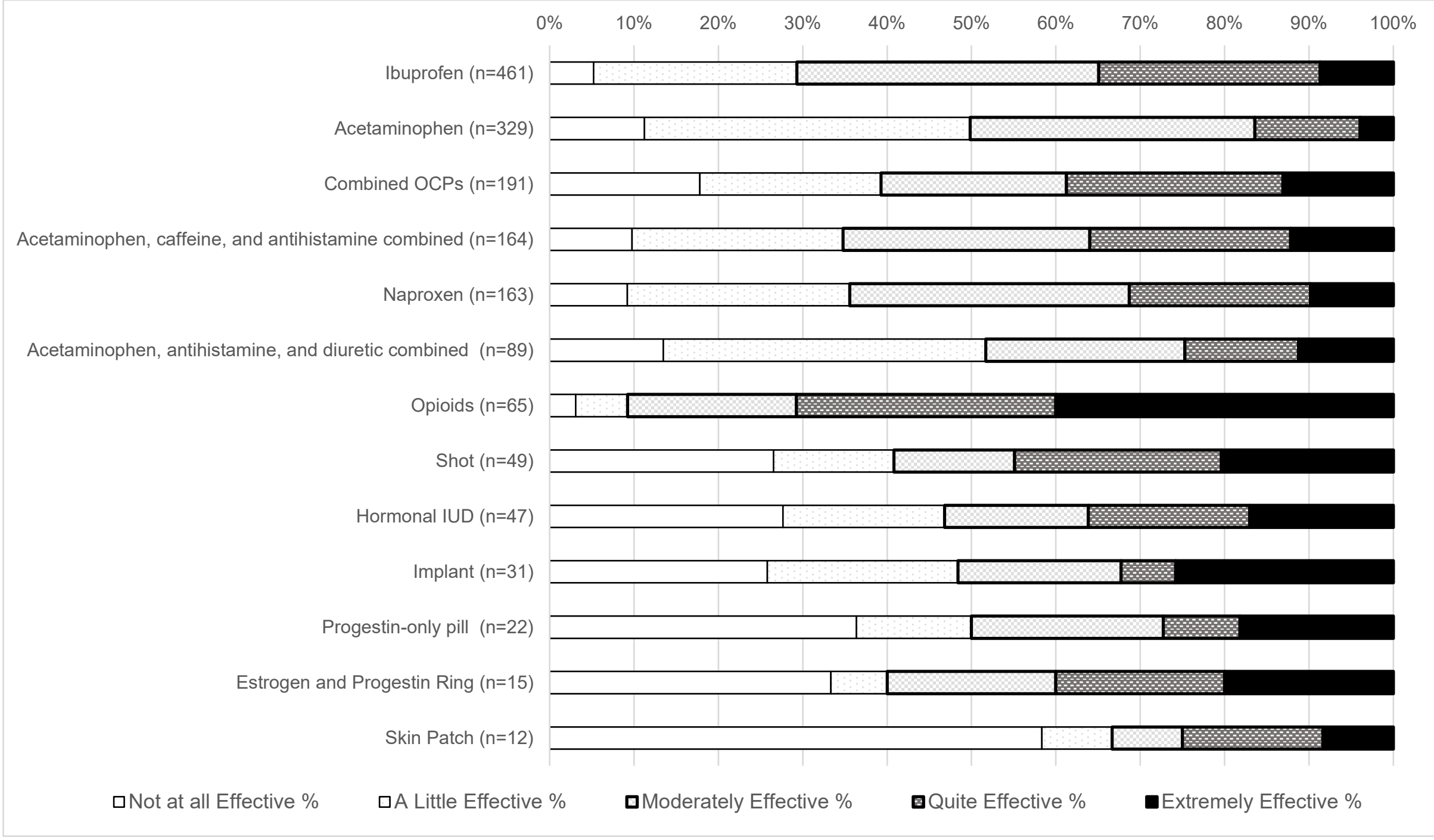

Treatments are ordered based on the frequency of use. 
Table 1. Perceiving Treatment as Ineffective by Dysmenorrhea Symptom-based Phenotypes (n=678)

\section{Multiple Severe}

Treatments ${ }^{1}$ (\# Users)

$\begin{array}{cc}\text { Mild Localized } & \text { Severe Localized } \\ \text { Pain Phenotype } & \text { Pain Phenotype } \\ \text { n (\%) } & \text { n (\%) }\end{array}$

Symptoms

Phenotype $\quad \mathbf{p}^{2}$

n (\%)

\begin{tabular}{|c|c|c|c|c|}
\hline Ibuprofen $(n=461)$ & $32(19.39 \%)$ & $57(34.34 \%)$ & $46(35.38 \%)$ & 0.0018 \\
\hline Acetaminophen $(n=329)$ & $46(41.44 \%)$ & $70(56.45 \%)$ & $48(51.06 \%)$ & 0.0682 \\
\hline Combined OCP $(n=191)$ & $15(23.44 \%)$ & $40(52.63 \%)$ & $20(39.22 \%)$ & 0.0019 \\
\hline Acetaminophen, caffeine, and & $11(22 \%)$ & $24(34.29 \%)$ & $22(50 \%)$ & 0.0173 \\
\hline \multicolumn{5}{|l|}{ antihistamine combined $(n=164)$} \\
\hline Naproxen $(n=163)$ & $11(23.91 \%)$ & $26(38.81 \%)$ & $21(42 \%)$ & 0.1452 \\
\hline Acetaminophen, antihistamine, & $11(47.83 \%)$ & $17(44.74 \%)$ & $18(64.29 \%)$ & 0.2681 \\
\hline \multicolumn{5}{|l|}{ and diuretic combined $(n=89)$} \\
\hline Opioids $(n=65)$ & $1(12.5 \%)$ & $4(13.79 \%)$ & $1(3.57 \%)$ & 0.3831 \\
\hline Hormonal IUD $(n=47)$ & $2(16.67 \%)$ & $11(55 \%)$ & $9(60 \%)$ & 0.0632 \\
\hline Shot $(n=49)$ & $3(27.27 \%)$ & $7(35 \%)$ & $10(55.56 \%)$ & 1.0000 \\
\hline Implant $(n=31)$ & $4(57.14 \%)$ & $8(61.54 \%)$ & $3(27.27 \%)$ & 0.2438 \\
\hline Progestin-only pill $(n=22)$ & $3(33.33 \%)$ & $4(57.14 \%)$ & $4(66.67 \%)$ & 0.5624 \\
\hline Estrogen-Progestin Ring $(n=15)$ & $1(25 \%)$ & $4(50 \%)$ & $1(33.33 \%)$ & 0.7986 \\
\hline Patch $(n=12)$ & $1(100 \%)$ & $5(62.5 \%)$ & $2(66.67 \%)$ & 0.5758 \\
\hline
\end{tabular}

${ }^{1}$ Treatments are ordered based on the frequency of use.

${ }^{2}$ Pvalue is based on comparison between perceived effective and perceived ineffective across three symptom-based phenotypes using Fisher's Exact Test; Bolded: $p<.05$

OCP: Oral contraceptive pills. IUD: Intrauterine device. 
Table 2. Correlates of Perceived Treatment Ineffectiveness for Ibuprofen $(n=461)$

\begin{tabular}{|c|c|c|c|c|c|c|c|c|c|c|}
\hline \multirow[b]{2}{*}{ Variable (reference) } & \multicolumn{5}{|c|}{ Bivariate Logistic Regression } & \multicolumn{5}{|c|}{ Multiple Logistic Regression ${ }^{1}$} \\
\hline & Beta & SE & OR & $95 \% \mathrm{Cl}$ & $\mathbf{p}$ & Beta & SE & OR & $95 \% \mathrm{Cl}$ & $\mathbf{p}$ \\
\hline \multicolumn{11}{|c|}{ Dysmenorrhea Symptom-Based Phenotypes } \\
\hline Severe Localized Pain (Mild) & 0.78 & 0.26 & 2.17 & {$[1.32-3.59]$} & 0.00 & 0.65 & 0.27 & 1.92 & {$[1.13-3.25]$} & 0.01 \\
\hline Severe Multiple Symptoms (Mild) & 0.82 & 0.27 & 2.28 & [1.34-3.85] & 0.00 & 0.51 & 0.29 & 1.67 & {$[0.94-2.94]$} & 0.08 \\
\hline \multicolumn{11}{|l|}{ Demographics } \\
\hline Age & 0.03 & 0.01 & 1.03 & {$[1.00-1.05]$} & 0.06 & & & & & \\
\hline Ethnicity-Hispanic & -0.15 & 0.31 & 0.86 & {$[0.47-1.59]$} & 0.64 & -0.08 & 0.33 & 0.92 & {$[0.48-1.76]$} & 0.81 \\
\hline Race Asian (White) & -0.30 & 0.48 & 0.74 & {$[0.29-1.91]$} & 0.53 & & & & & \\
\hline Black (White) & -0.44 & 0.34 & 0.65 & {$[0.34-1.25]$} & 0.20 & & & & & \\
\hline Other (White) & 0.30 & 0.31 & 1.35 & {$[0.73-2.48]$} & 0.34 & & & & & \\
\hline \multicolumn{11}{|l|}{ Clinical } \\
\hline Number of chronic pain conditions & 0.30 & 0.07 & 1.35 & {$[1.18-1.55]$} & 0.00 & 0.23 & 0.08 & 1.26 & {$[1.08-1.47]$} & 0.00 \\
\hline Comorbid gynecological condition & 0.38 & 0.22 & 1.46 & {$[0.95-2.26]$} & 0.09 & 0.16 & 0.24 & 1.17 & {$[0.79-1.88]$} & 0.49 \\
\hline \multicolumn{11}{|l|}{ Psycho-behavioral } \\
\hline Pain Catastrophizing & 0.02 & 0.01 & 1.02 & {$[1.01-1.04]$} & 0.01 & 0.02 & 0.01 & 1.02 & {$[1.00-1.04]$} & 0.07 \\
\hline Perceived Stress & 0.00 & 0.02 & 1.00 & [0.97-1.03] & 0.93 & -0.04 & 0.02 & 0.96 & {$[0.92-1.00]$} & 0.05 \\
\hline Anxiety & 0.02 & 0.01 & 1.02 & [0.99-1.04] & 0.22 & & & & & \\
\hline Depression & 0.02 & 0.01 & 1.02 & [1.00-1.04] & 0.11 & & & & & \\
\hline Sleep disturbance & -0.00 & 0.03 & 0.99 & {$[0.95-1.05]$} & 0.98 & & & & & \\
\hline
\end{tabular}

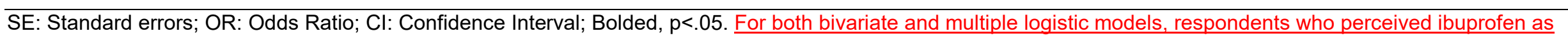
effective were the outcome reference group.

${ }^{1}$ The covariates for the multiple logistic regression model were selected by fitting all possible combinations of covariate variables and selecting the model with the lowest Akaike's Information Criteria; Pseudo R2: Nagelkerke $R^{2}=0.12$, McKelveyZavoina $R^{2}=0.09$, McFadden $R^{2}=0.07$ 
Table 3. Correlates of Perceived Treatment Ineffectiveness for Acetaminophen $(n=329)$

\section{Bivariate Logistic Regression}

Multiple Logistic Regression ${ }^{1}$

\begin{tabular}{|c|c|c|c|c|c|c|c|c|c|c|}
\hline \multirow{2}{*}{ Variable (reference) } & & & & & & & & & & \\
\hline & Beta & SE & OR & $95 \% \mathrm{Cl}$ & $\mathbf{p}$ & Beta & SE & OR & $95 \% \mathrm{Cl}$ & $\mathbf{p}$ \\
\hline \multicolumn{11}{|c|}{ Dysmenorrhea Symptom-Based Phenotypes } \\
\hline Severe Localized Pain (Mild) & 0.61 & 0.26 & 1.83 & & 0.02 & 0.54 & 0.27 & 1.72 & & $<.05$ \\
\hline Severe Multiple Symptoms (Mild) & 0.39 & 0.28 & 1.47 & & 0.17 & 0.30 & 0.30 & 1.35 & & 0.32 \\
\hline \multicolumn{11}{|l|}{ Demographics } \\
\hline Age & 0.03 & 0.02 & 1.03 & & 0.04 & & & & & \\
\hline Ethnicity-Hispanic & -0.28 & 0.34 & 0.76 & & 0.42 & -0.23 & 0.36 & 0.79 & & 0.51 \\
\hline Race Asian (White) & -1.33 & 0.58 & 0.27 & & 0.02 & & & & & \\
\hline Black (White) & -0.23 & 0.35 & 0.80 & & 0.51 & & & & & \\
\hline Other (White) & 0.11 & 0.35 & 1.11 & & 0.77 & & & & & \\
\hline \multicolumn{11}{|l|}{ Clinical } \\
\hline Number of chronic pain conditions & 0.19 & 0.08 & 1.21 & & 0.01 & 0.19 & 0.08 & 1.21 & & 0.03 \\
\hline Comorbid gynecological condition & 0.35 & 0.24 & 1.42 & & 0.14 & & & & & \\
\hline Psycho-behavioral & & & & & & 0.26 & 0.25 & 1.30 & & 0.30 \\
\hline Pain Catastrophizing & 0.01 & 0.01 & 1.01 & & $<0.50$ & & & & & \\
\hline Perceived Stress & 0.01 & 0.02 & 1.01 & & 0.73 & & & & & \\
\hline Anxiety & 0.01 & 0.01 & 1.01 & & 0.45 & & & & & \\
\hline Depression & 0.02 & 0.01 & 1.02 & & 0.16 & & & & & \\
\hline Sleep disturbance & -0.03 & 0.03 & 0.97 & & 0.23 & & & & & \\
\hline
\end{tabular}

SE: Standard errors; OR: Odds Ratio; Cl: Confidence Interval; Bolded, $p<.05$. For both bivariate and multiple logistic models, respondents who perceived acetaminophen as effective were the outcome reference group.

${ }^{1}$ The covariates for the multiple logistic regression model were selected by fitting all possible combinations of covariate variables and selecting the model with the lowest Akaike's Information Criteria; Pseudo $R^{2}$ : Nagelkerke $R^{2}=0.10$, McKelveyZavoina $R^{2}=0.06$, McFadden $R^{2}=0.05$. 
Table 4 Correlates of Perceived Treatment Ineffectiveness for Combined Oral Contraceptive Pills (n=191)

\begin{tabular}{|c|c|c|c|c|c|c|c|c|c|c|}
\hline \multirow[b]{2}{*}{ Variable (reference) } & \multicolumn{5}{|c|}{ Bivariate Logistic Regression } & \multicolumn{5}{|c|}{ Multiple Logistic Regression ${ }^{1}$} \\
\hline & Beta & SE & OR & $95 \% \mathrm{Cl}$ & $\mathbf{p}$ & Beta & SE & OR & $95 \% \mathrm{Cl}$ & $\mathbf{p}$ \\
\hline \multicolumn{11}{|c|}{ Dysmenorrhea Symptom-Based Phenotypes } \\
\hline Severe Localized Pain (Mild) & 1.29 & 0.37 & 3.63 & [1.74-7.55] & 0.00 & 1.24 & 0.40 & 3.46 & [1.58-7.57] & 0.00 \\
\hline Severe Multiple Symptoms (Mild) & 0.75 & 0.41 & 2.11 & [0.94-4.73] & 0.07 & 0.57 & 0.44 & 1.77 & {$[0.75-4.19]$} & 0.20 \\
\hline \multicolumn{11}{|l|}{ Demographics } \\
\hline Age & 0.03 & 0.02 & 1.03 & {$[0.99-1.07]$} & 0.16 & & & & & \\
\hline Ethnicity-Hispanic & -0.11 & 0.50 & 0.89 & {$[0.33-2.38]$} & 0.82 & & & & & \\
\hline Race Asian (White) & 1.13 & 0.88 & 3.08 & {$[0.55-17.34]$} & 0.20 & & & & & \\
\hline Black (White) & -0.17 & 0.53 & 0.84 & {$[0.30-2.39]$} & 0.75 & & & & & \\
\hline Other (White) & -0.38 & 0.62 & 0.69 & {$[0.20-2.32]$} & 0.54 & & & & & \\
\hline \multicolumn{11}{|l|}{ Clinical } \\
\hline Number of chronic pain conditions & 0.20 & 0.09 & 1.22 & {$[1.02-1.47]$} & 0.03 & & & & & \\
\hline Comorbid gynecological condition & 0.96 & 0.31 & 2.61 & {$[1.43-4.78]$} & 0.00 & 0.94 & 0.33 & 2.56 & [1.34-4.89] & 0.00 \\
\hline \multicolumn{11}{|l|}{ Psycho-behavioral } \\
\hline Pain Catastrophizing & 0.02 & 0.01 & 1.02 & {$[1.00-1.05]$} & 0.05 & & & & & \\
\hline Perceived Stress & 0.01 & 0.03 & 1.01 & {$[0.97-1.06]$} & 0.60 & & & & & \\
\hline Anxiety & 0.01 & 0.02 & 1.01 & {$[0.97-1.04]$} & 0.73 & -0.06 & 0.03 & 0.94 & {$[0.89-1.00]$} & 0.03 \\
\hline Depression & 0.03 & 0.02 & 1.04 & {$[1.00-1.07]$} & 0.05 & 0.06 & 0.03 & 1.06 & {$[1.00-1.13]$} & 0.04 \\
\hline Sleep disturbance & 0.01 & 0.03 & 1.01 & {$[0.95-1.07]$} & 0.86 & & & & & \\
\hline
\end{tabular}

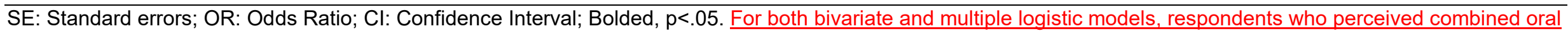
contraceptive pills as effective were the outcome reference group.

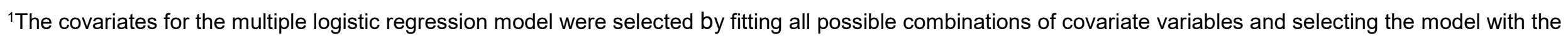
lowest Akaike's Information Criteria; Pseudo $\mathrm{R}^{2}$ : Nagelkerke $\mathrm{R}^{2}=0.18$, McKelveyZavoina $\mathrm{R}^{2}=0.17, \mathrm{McF}$ adden $\mathrm{R}^{2}=0.11$. 